\title{
Coût hospitalier des pathologies invasives à pneumocoque chez les enfants âgés de moins de 15 ans en Tunisie
}

Arwa Ben Salah, ${ }^{1,2}$ Sana El Mhamdi, ${ }^{1,2}$ Manel Ben Fredj, ${ }^{1}$ Chebil Ben Meriem, ${ }^{2,3}$ Jihen Bouguila, ${ }^{4}$ Khaled Ben Helel, ${ }^{5}$ Lamia Sfaihi, ${ }^{6}$ Rafiaa Mustapha, ${ }^{7}$ Mohamed Sfar ${ }^{8}$ et Mohamed Soltani ${ }^{1,2}$

${ }^{1}$ Service de Médecine préventive et d'Épidémiologie, Centre hospitalier universitaire (CHU), Monastir (Tunisie). ${ }^{2}$ Laboratoire de recherche, Épidémiologie appliquée à la santé de la mère et de l'enfant, CHU Monastir, Tunisie. ${ }^{3}$ Service de Pédiatrie, CHU, Monastir, Tunisie. ${ }^{4}$ Service de Pédiatrie, CHU Farhat Hached, Sousse, Tunisie. ${ }^{5}$ Service de Pédiatrie, CHU, Kairouan, Tunisie. ${ }^{6}$ Service de Pédiatrie, CHU Sfax, Tunisie. ${ }^{7}$ Service de Pédiatrie, Hôpital régional de Nabeul, Tunisie. ${ }^{8}$ Service de Pédiatrie, CHU, Mahdia, Tunisie. (Correspondance à Arwa Ben Salah à : bensalah.arwa@yahoo.fr ).

\section{Résumé}

Contexte : Les infections à pneumocoque sont une cause importante de morbidité et de mortalité dans le monde et en Tunisie. Des données sur le fardeau économique de ces infections sont nécessaires pour éclairer la décision d'inclure le vaccin antipneumococcique dans la vaccination systématique des enfants.

Objectifs : La présente étude visait à estimer le coût médical des hospitalisations causées par une pneumococcie invasive (pneumonie et méningite) chez les enfants de moins de 15 ans en Tunisie.

Méthode : Une étude prospective multicentrique a été menée dans 15 services de pédiatrie, dans différentes zones socio-économiques de la Tunisie, de juin 2014 à mai 2015. Tous les enfants de moins de 15 ans hospitalisés pour une pneumonie à pneumocoque ou une méningite bactérienne confirmée ont été inclus. Un formulaire de rapport de cas a été rempli pour chaque cas éligible. La méthode des coûts à base d'activités (méthode $\mathrm{ABC}$ ) a été utilisée pour estimer le coût hospitalier. La saisie des données et l'analyse statistique ont été effectuées à l'aide du logiciel SPSS 20.0.

Résultats : Au cours de la période de l'étude, 727 enfants ont été hospitalisés pour une pneumonie à pneumocoque et 60 autres pour une méningite bactérienne, dont $21(35 \%)$ avaient une méningite à pneumocoque confirmée. Le coût hospitalier médian pour la pneumonie à pneumocoque était de TND 353, 910 et de TND 1680, 632 pour la méningite à pneumocoque. Par extrapolation globale des données, nous avons estimé que près de 1091 hospitalisations pour pneumonie à pneumocoque et 69 hospitalisations pour méningite à pneumocoque surviennent chaque année chez des enfants tunisiens de moins de 15 ans, pour un coût total de TND 502 079, 408.

Conclusion : Le fardeau économique des infections à pneumocoque semble être important en Tunisie. Les coûts estimés ne reflètent pas les coûts réels de cette infection. Des études sur le rapport coût-efficacité seraient utiles pour aider les responsables de l'élaboration des politiques à prendre les décisions nécessaires.

Mots clés : Pneumonie à pneumocoque ; méningite à pneumocoque; coût hospitalier ; Tunisie

Citation : Ben Salah A; El Mhamdi S; Ben Fredj M; Ben Meriem C; Bouguila J; Ben Helel K; et al. Coût hospitalier des pathologies invasives à pneumocoque chez les enfants âgés de moins de 15 ans en Tunisie. East Mediterr Health J. 2018;25(12):861-871. https://doi.org/10.26719/ emhj.19.036

Reçu : 22/12/16; accepté : 27/03/18

(c) Organisation mondiale de la Santé 2019. Certains droits réservés. La présente publication est disponible sous la licence Creative Commons Attribution-Pas d'utilisation commerciale - Partage dans les mêmes conditions 3.0 IGO (CC BY-NC-SA 3.0 IGO ; (https://creativecommons.org/ licenses/by-nc-sa/3.0/igo).

\section{Introduction}

Le pneumocoque constitue l'un des principaux germes responsables de pneumonie, de méningite et de septicémie chez les enfants (1). Les infections causées par le pneumocoque Streptococcus pneumoniae constituent une cause importante de morbidité et de mortalité partout dans le monde. Chaque année, 826000 enfants âgés de moins de cinq ans décèdent suite à une infection par le pneumocoque, ce qui correspond à $11 \%$ de tous les décès dans ce groupe d'âge (1). Les progrès réalisés en matière de réanimation et de prise en charge diagnostique et thérapeutique ont certes contribué à la réduction de cette mortalité mais ceci aux dépens d'une charge morbide plus importante, et par conséquent d'un coût plus élevé.

Dans sa note de synthèse de 2012, l'Organisation mondiale de la Santé (OMS) a recommandé l'introduction des vaccins antipneumococciques conjugués dans les programmes nationaux de vaccination des différents pays du monde (2). Deux vaccins conjugués (10-valent et 13-valent), ayant démontré leur innocuité et efficacité (3-8), sont actuellement disponibles et utilisés dans plus de 100 pays dans le monde entier, dont des pays à revenu faible ou intermédiaire (9). En outre, ces vaccins peuvent être facilement intégrés dans le Programme élargi de vaccination et co-administrés avec d'autres vaccins sans aucun risque d'interaction (2). Toutefois, le processus décisionnel doit se baser sur des données locales et des estimations objectives de la charge morbide et du coût de prise en charge des infections causées par le pneumocoque.

En Tunisie, les données disponibles montrent que ces infections constituent un problème réel de santé publique (10). En effet, l'étude réalisée par Khalifa et al. au Centre hospitalier universitaire (CHU) de Monastir en Tunisie a montré que $S$. pneumoniae était le germe le plus retrouvé dans les méningites des individus âgés de cinq ans et plus (11). De même, une étude communautaire réalisée par Maalej et al. a montré que S. pneumoniae 
occupait la deuxième position dans les méningites du nourrisson et du petit enfant et la première position dans les méningites de l'enfant de plus de cinq ans (12). La même étude a montré que ce germe se caractérisait par des taux importants de résistance à la pénicilline. Cependant, l'ampleur de ce problème et de sa charge réelle sur le plan des dépenses de santé n'a pas été précisée, d'où l'intérêt de la présente étude.

L'objectif de cette étude était d'estimer le coût hospitalier des méningites et des pneumonies à pneumocoque chez les enfants âgés de moins de 15 ans en Tunisie.

\section{Méthodes}

\section{Type, lieu et population d'étude}

Il s'agit d'une étude longitudinale prospective multicentrique réalisée entre le $1^{\text {er }}$ juin 2014 et le 31 mai 2015 dans 15 services de pédiatrie, au niveau de huit centres hospitalo-universitaires et trois hôpitaux régionaux, répartis dans différentes régions socio-économiques du pays : district de Tunis (trois sites), régions du nord-ouest (deux sites), du nord-est(trois sites), du centre-est (trois sites), du centre-ouest (un site), du sud-ouest (un site) et du sud-est (trois sites).

Notre population d'étude était constituée de l'ensemble des enfants âgés de moins de 15 ans, hospitalisés dans l'un des sites de l'étude pour pneumonie ou pleuropneumonie à pneumocoque ou méningite bactérienne. Dans cette étude, nous avons exclu les otites à pneumocoque étant donné qu'elles sont souvent traitées en ambulatoire. Nous avons également exclu les septicémies à pneumocoque en raison de la difficulté d'obtention d'une confirmation bactériologique.

Le diagnostic de pneumonie ou pleuropneumonie à pneumocoque était basé sur un ensemble de critères cliniques (fièvre, toux, douleur thoracique, dyspnée, râles crépitants localisés, souffle tubaire, etc.), radiologiques (opacité systématisée pouvant être associée à un épanchement pleural, ou pleurésie sans foyer de pneumonie) et biologiques (hyperleucocytose à polynucléose, $\mathrm{CRP}$ [C-reactive protein - protéine $\mathrm{C}$ réactive] supérieure à $20 \mathrm{mg} / \mathrm{L}$ et isolement de $S$. pneumoniae à l'hémoculture ou à la culture du liquide pleural). C'est ce dernier critère bactériologique qui permet de confirmer le diagnostic. Toutefois, étant donné que le germe n'est isolé par hémoculture que dans moins de $8 \%$ des cas (13), nous avons inclus dans cette étude les cas « probables », définis comme étant toutes les situations répondant aux critères cliniques, radiologiques et biologiques sans confirmation bactériologique avec une évolution favorable du patient sous antibiothérapie visant $S$. pneumoniae.

Le diagnostic de méningite bactérienne repose aussi sur un ensemble de critères cliniques (apparition soudaine de fièvre, refus de téter, vomissements, troubles de la conscience, convulsions, hypotonie, fontanelle bombée, céphalée, raideur de la nuque, etc.), de critères biologiques (examen cytobactériologique du liquide céphalo-rachidien: protéinorachie supérieure à $100 \mathrm{mg} / \mathrm{dL}$, glucorachie inférieure à $50 \%$ de la glycémie, pléiocytose à prédominance neutrophile, et identification du germe par examen direct et/ou culture et/ou recherche d'antigène $[\mathrm{Ag}]$ soluble). La confirmation du diagnostic se fait grâce au dernier critère biologique (identification du germe). Ainsi, le cas de méningite est classé en méningite bactérienne à pneumocoque confirmée, méningite bactérienne confirmée à d'autres germes ou méningite bactérienne à germe non identifié. Ce dernier groupe correspond aux cas probables de méningites bactériennes devant les signes cliniques évocateurs et l'examen cytologique du liquide céphalorachidien (protéinorachie élevée avec glucorachie diminuée et pléiocytose à prédominance neutrophile) sans confirmation bactériologique par identification du germe en cause. Ils correspondent aux cas de méningites décapitées par une antibiothérapie avant la pratique de la ponction lombaire.

Il convient de noter que les enfants dont le lieu de résidence n'appartient pas aux gouvernorats de l'étude étaient exclus de l'étude. Les méningites néonatales étaient également exclues.

\section{Déroulement de la surveillance et collecte des données}

Les cas éligibles de chaque site ont été repérés par un médecin coordonnateur de l'enquête (désigné par le chef du service correspondant). Les différentes données sociodémographiques (date de naissance, sexe, lieu de résidence, service d'hospitalisation), ainsi que les données cliniques, biologiques et radiologiques ont été consignées sur une fiche de renseignement spécifique pour chaque type d'infection (pneumonie ou méningite). Ces enfants ont été suivis tout au long de l'hospitalisation afin de notifier les différents soins procurés et médicaments consommés ainsi que l'évolution clinique et la durée totale de séjour.

\section{Calcul du coût}

\section{Méthode de calcul}

Pour les besoins de cette étude, nous avons identifié la prise en charge hospitalière des méningites bactériennes et des pneumonies à pneumocoque comme un « service » fourni aux malades et ayant un coût de revient. Ce coût a été par la suite calculé selon la méthode basée sur les activités - méthode ABC (Activity Based Costing). Il s'agit d'une méthode de calcul du coût, issue du domaine industriel (14), qui repose sur une vision transversale de l'entreprise (ici l'hôpital), c'est-à-dire une vision par processus où le concept d'activité joue un rôle central. Partant du constat que ce sont les activités qui consomment les ressources, et que les produits ou services consomment des activités, la méthode $A B C$ se propose, grâce à la description des activités qui sont effectivement réalisées, de comprendre comment les ressources sont effectivement consommées (15). Ainsi le processus de prise en charge hospitalière des méningites 


\section{Figure 1 Processus de prise en charge d'un enfant admis pour méningite bactérienne ou pneumonie à pneumocoque}

\section{Activités d'hébergement}

Hôtellerie, restauration, matériel et équipement multi-usage, élecricité, etc.

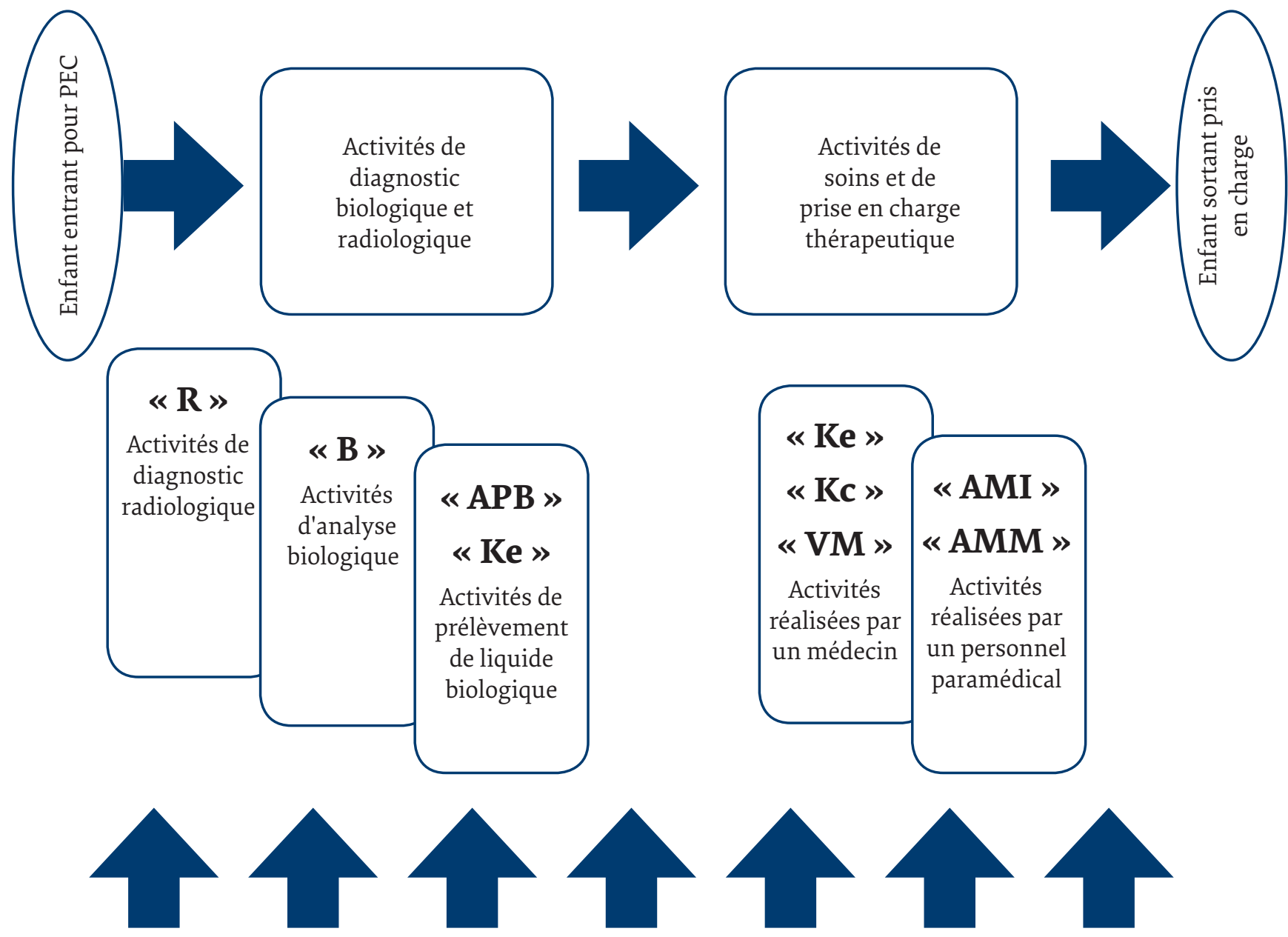

Ressources : électricité, réactifs, dispositifs médicaux, personnel médical et paramédical, médicaments produits pharmaceutiques, bâtiments, etc.

PEC : prise en charge

bactériennes et des pneumonies à pneumocoque consomme différentes ressources qui peuvent être regroupées en trois grandes catégories, selon les activités produites : les ressources utilisées dans les activités de diagnostic biologique et radiologique; les ressources utilisées dans les activités de soins et de prise en charge thérapeutique ; et les ressources utilisées lors des activités d'hébergement (Figure 1).

\section{Calcul proprement dit}

- Coût des activités de diagnostic biologique et radiologique

- Pour chaque patient, nous avons listé l'ensemble des activités de prélèvement de liquide biologique, d'analyse biologique et de diagnostic radiologique.
Ces activités ont été par la suite converties, selon une pondération prédéfinie par le ministère de la Santé, en un nombre d'APB (pour les Activités de Prélèvement de liquide biologique réalisées par un infirmier), de Ke (pour les activités de prélèvement de liquide biologique réalisées par un médecin), de (pour les tests biologiques réalisés aux laboratoires de biologie) et de $\mathrm{R}$ (pour les examens Radiologiques réalisés au service de radiologie) (16). En l'absence de données locales spécifiques pour chaque établissement, les coûts unitaires des APB, Ke, B et R utilisés dans cette étude étaient ceux élaborés par le ministère tunisien de la Santé, selon la méthode $A B C$, et utilisés actuellement pour la tarification des actes de soins : $\mathrm{B}=0,16$ dinar tunisien (TND) (USD 0,08); 
$\mathrm{R}=\mathrm{TND} 0,9$ (USD 0,46); $\mathrm{APB}=\mathrm{TND} 0,8$ (USD 0,41); $\mathrm{Ke}=\mathrm{TND} 1,2(\mathrm{USD} 0,61)$ (17). Le taux de change utilisé pour la conversion du dinar tunisien (TND) au dollar des États-Unis (USD) était celui moyen de l'année 2015: 1 TND = 0,51 USD. Ainsi, pour chaque patient, le coût total des activités de prélèvement de liquide biologique, des activités d'analyse biologique et de diagnostic radiologique était calculé en multipliant le nombre d'APB, de Ke, de B et de R consommés par leurs coûts unitaires respectifs.

- Coût des activités de soins et de prise en charge thérapeutique

- Les différentes activités médicales et paramédicales ont été également listées puis converties en un nombre d'AMI (Activités de soins réalisées par un infirmier), d'AMM (Activités de soins réalisées par un technicien de physiothérapie), de Ke (actes spécialisés réalisés par un médecin et Kc (actes chirurgicaux réalisés par un médecin) $($ avec AMI $=0,5$ TND [USD 0,25]; AMM $=$ TND 0,6 [USD 0,31]; de Ke $=1,2$ TND [USD 0,61] et $1 \mathrm{Kc}=1,4 \mathrm{TND}$ [USD 0,71]). Pour les visites médicales de routine (VM), un nombre moyen de deux visites par jour d'une durée de 15 minutes chacune a été comptabilisé pour chaque patient; un coût moyen de 4 TND (USD 2) a été estimé par le service financier du CHU Fattouma Bourguiba de Monastir (en se référant au salaire annuel brut d'un médecin spécialiste en pédiatrie et le nombre d'heures travaillées par an) et a été utilisé pour l'ensemble des patients. Ainsi, le coût total de chaque activité était calculé pour chaque patient en multipliant le nombre d'AMI, d'AMM, de Ke, de Kc et de VM consommés par leurs coûts unitaires respectifs. Les dispositifs médicaux utilisés pour les soins et l'administration des médicaments (seringues, branules, perfuseurs, etc.) ainsi que les produits pharmaceutiques (solutés de remplissage, électrolytes, etc.) et médicaments (antibiotiques, antipyrétiques, etc.) consommés par chaque patient étaient également listés. Leurs coûts étaient calculés en multipliant le nombre d'unités consommées par le prix unitaire de chaque produit (prix d'achat de la pharmacie centrale ou prix du marché).

- Coût des activités d'hébergement : coût standard

- Le coût des activités d'hébergement incluant la restauration, la blanchisserie, l'électricité, l'amortissement des bâtiments et des dispositifs et matériels à usage multiple a également été pris en compte. Le coût total était estimé par la multiplication du nombre des journées d'hébergement par le coût unitaire d'une journée. Ce coût unitaire a été estimé par le service financier du CHU Fattouma Bourguiba de Monastir pour chaque catégorie de services: TND 40 (USD 20,4) pour le service de pédiatrie, 170 TND (USD 86,7) pour les unités de soins intensifs pédiatriques et TND 55 (USD 28) pour le service de chirurgie pédiatrique. Ces coûts étaient calculés en divisant les dépenses annuelles du service correspondant par le nombre de journées d'hospitalisation au cours de cette même année.

\section{Estimation du taux des méningites à pneumocoque}

Dans le but d'avoir une estimation plus correcte de ce taux, il a fallu inclure dans le numérateur le nombre de méningites dues probablement au pneumocoque. Ce nombre était estimé, dans chaque site, par la multiplication du nombre de méningites bactériennes à germe non identifié par la proportion des méningites à pneumocoque parmi les méningites bactériennes confirmées.

\section{Analyse des données}

L'ensemble des fiches de collecte des données remplies ont été acheminées au service de médecine préventive et d'épidémiologie du CHU de Monastir. Àce niveau, la saisie des données ainsi que leur analyse ont été effectuées par le logiciel SPSS 20.0. Le taux d'incidence (TI) est défini par le nombre de nouveaux cas d'une maladie $(\mathrm{m})$ apparus pendant une durée donnée $(\Delta t)$, rapporté au nombre de sujets à risque pendant la période considérée (18). Le TI était calculé pour les gouvernorats où le service de pédiatrie, inclus dans l'étude, est le seul service de pédiatrie qui hospitalise les cas de pneumonie et de méningite dans le gouvernorat, soit 10 gouvernorats. Le nombre de sujets à risque de chaque gouvernorat était estimé grâce aux données démographiques fournies par l'Institut National de la Statistique (19).

\section{Résultats}

\section{Pneumonies/pleuropneumonies à pneumocoque}

\section{Caractéristiques de la population étudiée}

Entre juin 2014 et mai 2015, 727 enfants âgés de moins de 15 ans étaient hospitalisés pour pneumonie ou pleuropneumonie à pneumocoque dans les 15 services de pédiatrie inclus dans l'étude. L'âge médian des patients était de 17,3 mois (intervalle interquartile [IIQ] : 8,1-36) et leur sex ratio était de 1,33. La durée médiane de séjour était estimée à 4 jours (IIQ : 3 - 7) (Tableau 1).

\section{Estimation du taux d'incidence hospitalière des pneumonies/ pleuropneumonies à pneumocoque}

Le taux d'incidence hospitalière global des pneumonies/ pleuropneumonie à pneumocoque chez les enfants de moins de 15 ans dans les 10 gouvernorats a été estimé à 41,3 cas/100 000 enfants.

\section{Estimation du coût hospitalier des pneumonies/ pleuropneumonies à pneumocoque}

Le coût hospitalier médian était de 353,910 TND (IIQ : 269,209-538,100) [USD 180,5 (IIQ : 137,3-274,4)], avec un coût minimal de 73,500 TND (USD 37,5) et un coût maximal de 6713,542 TND (USD 3423,9). Le coût total occasionné par les hospitalisations 


\begin{tabular}{|c|c|}
\hline Variables & Nbre (\%) \\
\hline \multicolumn{2}{|l|}{ Sexe } \\
\hline Masculin & $415(57,1)$ \\
\hline Féminin & $312(42,9)$ \\
\hline \multicolumn{2}{|l|}{ Âge (mois) } \\
\hline $0-24$ & $460(63,3)$ \\
\hline $24-60$ & $175(24)$ \\
\hline $60-120$ & $68(9,4)$ \\
\hline $120-180$ & $24(3,3)$ \\
\hline \multicolumn{2}{|l|}{ Lieu d'hospitalisation } \\
\hline District de Tunis & $80(11)$ \\
\hline Nord-Ouest & $77(10,6)$ \\
\hline Nord-Est & $54(7,4)$ \\
\hline Centre-Ouest & $68(9,4)$ \\
\hline Centre-Est & $359(49,4)$ \\
\hline Sud-Ouest & $6(0,8)$ \\
\hline Sud-Est & $83(11,4)$ \\
\hline Antibiothérapie avant l'admission & $267(36,7)$ \\
\hline Admission en unité de soins intensifs & $17(2,3)$ \\
\hline \multicolumn{2}{|l|}{ Évolution clinique (n = 723) } \\
\hline $\begin{array}{l}\text { Amélioration } \\
\text { Survenue de complications }\end{array}$ & $\begin{array}{c}657(90,9) \\
66(9,1)\end{array}$ \\
\hline Procédures chirurgicales (drainage thoracique/thoracoscopie) $(n=723)$ & $10(1,4)$ \\
\hline \multicolumn{2}{|l|}{ État à la sortie } \\
\hline $\begin{array}{l}\text { Décès } \\
\text { Guérison sans séquelles } \\
\text { Guérison avec séquelles } \\
\text { Indéterminé }\end{array}$ & $\begin{array}{c}8(1,1) \\
704(96,8) \\
11(1,5) \\
4(0,6)\end{array}$ \\
\hline
\end{tabular}

pour pneumonies à pneumocoque était de TND 99 621,707 (USD 50 807,1), réparti comme suit: $47,8 \%$ pour les activités d'hébergement (médiane: TND 160; IIQ : 120-280 [USD 81,6; IIQ : 61,2-91,8]), $27,2 \%$ pour les activités de soins et de prise en charge thérapeutique (médiane :TND 76,583 ; IIQ : 56,378-122,743 [USD 39,1; IIQ: 28,7-62,6]) et $25 \%$ pour les activités de diagnostic (médiane: TND 105,800 ; IIQ : 79,800-140,600 [USD 54 ; IIQ : 40,771,7]) (Tableau 2).

\begin{tabular}{|c|c|c|c|}
\hline Coût (TND) & Médiane & IIQ & Min-Max \\
\hline Activités de diagnostic biologique et radiologique & 105,800 & $79,800-140,600$ & $0-393,600$ \\
\hline Tests biologiques & 58,400 & $44,800-80,00$ & \\
\hline Examens radiologiques & 45 & $22,500-67,500$ & $22-887,500$ \\
\hline Prélèvement des échantillons biologiques & 2,400 & $1,200-3,600$ & $0-74,400$ \\
\hline Activités de soins et de prise en charge thérapeutique & 76,583 & $56,378-122,743$ & $0-1606,931$ \\
\hline Médicaments et produits pharmaceutiques & 14,554 & $7,741-28,170$ & \\
\hline Dispositifs médicaux & 3,369 & $2,318-6,381$ & $0-227,314$ \\
\hline Soins médicaux spécialisés et visites de routine & 32 & 24-56 & $8-514$ \\
\hline Soins paramédicaux & 22,5 & $15-40,500$ & 3-811 \\
\hline Activités d'hébergement (coût standard) & 160 & $120-280$ & $40-3740$ \\
\hline
\end{tabular}

Dinar tunisien $(T N D)=$ USD 0,51 (moyenne pour l'année 2015); IIQ : intervalle interquartile. 


\section{Méningites à pneumocoque}

\section{Caractéristiques des cas de méningite à pneumocoque}

Au cours de la période d'étude, 60 enfants âgés entre un mois et 15 ans étaient hospitalisés pour une méningite bactérienne, dont $35 \%(n=21)$ l'étaient pour une méningite à pneumocoque. L'âge médian des enfants hospitalisés pour méningite à pneumocoque était de 5,3 mois (IIQ: 4,2-20,8). La durée médiane de séjour était de 16 jours (IIQ : 10,5-35,5). Sept enfants (33,33\%) étaient décédés et six ont gardé des séquelles (quatre patients ont gardé une épilepsie ; un patient a gardé une surdité et une paralysie faciale et un enfant a gardé une hydrocéphalie avec des lésions cérébrales) (Tableau 3).

\section{Estimation du taux d'incidence hospitalière des méningites à pneumocoque}

Le taux d'incidence hospitalière global des méningites à pneumocoque confirmées chez les enfants de moins de 15 ans dans les 10 gouvernorats a été estimé à 9/1 000000 enfants. Le taux d'incidence hospitalière global des méningites à pneumocoque confirmées et probables chez les enfants de moins de 15 ans dans les 10 gouvernorats a été estimé à 26,3/1 000000 enfants.

\section{Estimation du coût hospitalier des méningites à pneumocoque}

Le coût hospitalier médian était de TND 1680,632 (IIQ : 1437,666-3844,384) [USD 857,1 (IIQ: 733,2-1705,6)], avec un coût minimal de TND 325,380 (USD 165,9) et un coût maximal de TND 5700,398 (USD 2907,2). Le coût total occasionné par les hospitalisations pour méningites à pneumocoque était de TND 49506,959 (USD 25248,5), réparti comme suit : $46 \%$ pour les activités d'hébergement (médiane: TND 795; IIQ : 560-1800 [USD405,5; IIQ : 285,6-918]), $30 \%$ pour les activités de soins et de prise en charge thérapeutique (médiane : 558,845 TND ; IIQ: 396,272-1017,921 [USD 285; IIQ: 202,1-519,1]) et $24 \%$ pour les activités de diagnostic (médiane: TND 462,650; IIQ : 394,850-882,950 [USD 235,9 ; IIQ : 201,4-450,3]) (Tableau 4).

\section{Extrapolation des résultats}

L'extrapolation des données de cette étude à l'ensemble de la population tunisienne d'enfants âgés de moins de 15 ans $(n=2641917)$ nous a permis d'estimer à TND 502 079,408 (USD 256 060,5) le coût annuel total des hospitalisations occasionnées par ces deux maladies (Tableau 5).

\section{Discussion}

Cette étude nous a permis d'estimer le coût médical direct des cas hospitaliers de pneumonies et méningites à pneumocoque en Tunisie. C'est la première étude réalisée dans notre pays pour fournir aux décideurs des données locales fiables sur l'ampleur de ce problème et ses répercussions dans le but d'orienter leur décision quant à l'introduction du vaccin. En effet, l'évaluation économique fait partie des principaux éléments décisionnels pouvant aider à se prononcer sur l'intérêt d'introduire tel ou tel vaccin dans le calendrier national de vaccination.

La méthode de calcul du coût utilisée est celle basée sur les activités : méthode $\mathrm{ABC}$ (Activity Based Costing) initialement utilisée dans le milieu industriel puis transposée dans le domaine hospitalier (14). Cette approche favorise l'efficience et donc la performance du système de santé(20). Cette méthode nous a permis d'estimer à TND 353,910 (IIQ : 269,209-538,100 [USD 180,5 (IIQ: 137,3-274,4)] le coût médian de prise en charge hospitalière d'un épisode de pneumonie à pneumocoque et à TND 1680,632 (IIQ : 437,666-3844,384) [USD 857,1 (IIQ : 733,2-1705,6)] celui de méningite. En extrapolant ces chiffres au nombre

\begin{tabular}{|c|c|}
\hline \multicolumn{2}{|c|}{$\begin{array}{l}\text { Tableau } 3 \text { Caractéristiques sociodémographiques et cliniques } \\
\text { des enfants âgés de moins de } 15 \text { ans hospitalisés pour } \\
\text { méningite à pneumocoque }\end{array}$} \\
\hline Variables & Nbre (\%) \\
\hline \multicolumn{2}{|l|}{ Sexe } \\
\hline Masculin & $11(52,4)$ \\
\hline Féminin & $10(47,6)$ \\
\hline \multicolumn{2}{|l|}{ Âge (mois) } \\
\hline $0-24$ & $16(76,2)$ \\
\hline $24-60$ & $2(9,5)$ \\
\hline $60-120$ & $2(9,5)$ \\
\hline $120-180$ & $1(4,8)$ \\
\hline \multicolumn{2}{|l|}{ Lieu d'hospitalisation } \\
\hline District de Tunis & $8(38,1)$ \\
\hline Nord-ouest & $0(0)$ \\
\hline Nord-est & $1(4,8)$ \\
\hline Centre-ouest & $3(14,3)$ \\
\hline Centre-est & $7(33,4)$ \\
\hline Sud-ouest & $0(0)$ \\
\hline Sud-est & $2(9,5)$ \\
\hline Admission en unité de soins intensifs & $8(38,09)$ \\
\hline \multicolumn{2}{|l|}{ Évolution clinique } \\
\hline \multicolumn{2}{|l|}{ Amélioration } \\
\hline Choc septique & $4(19)$ \\
\hline Convulsion/état de mal épileptique & $3(15)$ \\
\hline Autres complications neurologiques & $11(55)$ \\
\hline $\begin{array}{l}\text { (méningo-encéphalite, ventriculite, } \\
\text { hydrocéphalie, thrombophlébite, etc.) }\end{array}$ & $10(50)$ \\
\hline Mastoïdite & $1(5)$ \\
\hline \multicolumn{2}{|l|}{ État à la sortie } \\
\hline Décès & $7(33,33)$ \\
\hline Guérison sans séquelles & $8(38,09)$ \\
\hline Guérison avec séquelles & $6(28,57)$ \\
\hline
\end{tabular}




\begin{tabular}{|c|c|c|c|}
\hline Coût (DT) & Médiane & IIQ & Min-Max \\
\hline Activités de diagnostic biologique et radiologique & 462,650 & $394,850-882,950$ & \\
\hline Tests biologiques & 214 & $138,800-284,400$ & $67,200-579,200$ \\
\hline Examens radiologiques & 315 & $162-516,350$ & $22,500-656,100$ \\
\hline Prélèvement des échantillons biologiques & 31,2 & $17,700-35,700$ & $10,800-58,800$ \\
\hline Activités de soins et de prise en charge thérapeutique & 558,845 & $396,272-1017,921$ & \\
\hline Médicaments et produits pharmaceutiques & 118,173 & $63,108-357,489$ & $11,959-1173,625$ \\
\hline Dispositifs médicaux & 40,232 & $27,864-76,802$ & $4,197-270,770$ \\
\hline Soins médicaux spécialisés et visites de routine & 134 & $109-260$ & $8-448$ \\
\hline Soins paramédicaux & 181,250 & $128-366,625$ & $14,500-1139,500$ \\
\hline Activités d'hébergement (coût standard) & 795 & $560-1800$ & $105-3150$ \\
\hline
\end{tabular}

Dinar tunisien $($ TND) = USD 0,51 (moyenne pour l'année 2015) ; IIQ : intervalle interquartile.

annuel de pneumonies et méningites à S.pneumoniae chez les enfants âgés de moins de 15 ans en Tunisie, le montant du coût direct occasionné par ces infections est estimé à près de TND 502 079,408, soit USD 256 060,5 un coût considérable dans un pays où le revenu mensuel moyen par habitant est de TND 544, soit USD 277,4 et les dépenses moyennes par personne et par an sont de TND 363 (USD 185,1) en 2015 (21,22). L'importance de ce coût devient plus tangible en le comparant au coût d'autres pathologies en Tunisie telles que le cancer. En effet, l'étude de Ben Gobrane et al. a montré que le coût de prise en charge du cancer invasif du col de l'utérus variait de TND 777 à 7458 (USD 396,3 - 3803,6) (23).

Dans la littérature, les données à propos du coût des infections invasives à pneumocoque étaient limitées à celles de quelques pays d'Europe et d'Amérique. Une comparaison directe avec les données disponibles semble difficile du fait des disparités existantes concernant aussi bien l'environnement socioéconomique que la méthodologie et la perspective utilisées pour le calcul du coût. En Espagne, le coût médian des hospitalisations dues aux pneumonies invasives à pneumocoque chez les enfants âgés de moins de 18 ans était estimé à 4533 euros (EUR) (IIQ : 1399 - 6188 [USD 5893 (IIQ : 1819-8044)]. La méthode ainsi utilisée pour le calcul du coût était celle basée sur «le groupe homogène de malades » (Diagnosis related group [DRG]) (24). Cette méthode a été aussi utilisée pour calculer le coût hospitalier des pneumonies et méningites à pneumocoque chez les sujets de moins de 20 ans en NouvelleZélande. Les coûts médians estimés pour ces deux infections étaient de NZD 2352 (USD 1678) et NZD 7116 (USD 5078), respectivement (25). En Italie, le coût annuel de prise en charge des enfants présentant une infection à pneumocoque était de EUR 59604477 (USD 56624 253), le coût était estimé dans une population pédiatrique trois fois plus nombreuse que celle de la Tunisie selon une perspective sociétale, incluant aussi bien le coût direct que le coût indirect (en rapport avec la perte de productivité), les cas hospitalisés et les cas traités en ambulatoire. Le coût direct représentait 39,9\% du coût total et était réparti comme suit : 60,6 \% pour le traitement des otites moyennes aiguës, 31,9\% pour les pneumonies (soit EUR 7586517 [USD 7207 191]), 6,5 \% pour les bactériémies et $1 \%$ pour les méningites (soit EUR 596045 [USD 566 243]) (26).

Une étude moins récente, réalisée aux États-Unis en 2004, a utilisé le modèle basé sur l'arbre de décision (decision tree-based model) pour calculer le coût total des infections à pneumocoque. Elle a inclus les différentes localisations de l'infection (méningites, pneumonies, otites, sinusites et bactériémies), les cas hospitaliers et ambulatoires ainsi que les différentes tranches d'âge (enfants, adultes et sujets âgés). Le coût médical direct estimé était de 3,7 milliards de dollars (USD), dont $17 \%$ occasionnés par la prise en charge d'enfants âgés de moins de 18 ans, soit

\begin{tabular}{|c|c|c|c|}
\hline & $\begin{array}{l}\text { Pneumonie à } \\
\text { pneumocoque }\end{array}$ & $\begin{array}{l}\text { Méningite à } \\
\text { pneumocoque }\end{array}$ & Total \\
\hline Nombre annuel d'hospitalisations & 1091 & 69 & 1160 \\
\hline Coût annuel occasionné par les hospitalisions (DT) & 386115,800 & 115963,608 & 502079,408 \\
\hline $\begin{array}{l}\text { Nombre annuel des séquelles liées aux cas } \\
\text { hospitaliers }\end{array}$ & 18 & 16 & 34 \\
\hline Nombre de décès annuels liés aux cas hospitaliers & 16 & 10 & 26 \\
\hline
\end{tabular}

Dinar tunisien $($ TND) = USD 0,51 (moyenne pour l'année 2015). 
USD 629000 000. Ce coût s'élève à 1,2 milliard de dollars si on considère le coût indirect lié à la perte de productivité suite au décès ou handicap généré par la maladie (27) un coût total calculé pour les 61027947 enfants vivants aux États-Unis (23 fois la population pédiatrique de la Tunisie) (28).

Les études réalisées en Amérique Latine ont mis en évidence le fait que le coût médical direct moyen des cas hospitaliers de pneumonies à pneumocoque variait selon les pays, entre USD 372 au Brésil et USD 1147 en Uruguay (29-31) et atteignant USD 1995 pour les cas compliqués d'empyème au Chili (31). Pour les cas de méningites, le coût médical direct moyen était estimé à USD 5666,43 au Brésil chez les enfants âgés de moins de 13 ans et à USD 2590,4 au Chili chez les enfants âgés de moins de trois ans. Une revue systématique de la littérature en Amérique latine a estimé un coût direct moyen de USD 2062 pour les cas hospitaliers de pneumonie à pneumocoque et de USD 4692 pour les cas de méningites (32). Des résultats différents d'un pays à l'autre qui, en l'absence d'études dans des pays de niveau de revenu comparable au nôtre, doivent être interprétés avec prudence, d'autant plus qu'ils sont issus de pays comme l'Italie, la Nouvelle-Zélande et l'Espagne dont le revenu national brut (RNB) par habitant est 8 fois plus important que celui de la Tunisie, et les États-Unis d'Amérique dont le RNB est 13 fois plus important (33).

Il existe deux autres points à noter: la perspective utilisée et, par la suite, les coûts inclus dans le calcul. Dans notre étude, nous nous sommes limités au calcul du coût médical direct selon une perspective du système de soins, qui est à son tour sous-estimé du fait que les coûts unitaires utilisés, proposés par le Ministère de la Santé, n'ont pas été mis à jour depuis 1996. Ainsi, nous n'avons pas inclus les coûts indirects, représentés par la perte de productivité en raison du décès prématuré ou de l'incapacité générée par la maladie et la perte de productivité des parents à cause de l'hospitalisation de leurs enfants.

De plus, uniquement l'épisode aigu menant à l'hospitalisation est pris en considération. Le coût des pneumonies traitées en ambulatoire n'était pas calculé, le coût a été estimé, pour un épisode, à $18 \%$ du coût de prise en charge d'un cas hospitalisé et peut atteindre 36\% pour les pneumonies sévères (34), de même que le coût de la prise en charge des séquelles, en particulier celles de la méningite, estimé à 3 à 5 fois le coût de l'épisode aigu (32). Dans cette étude, les autres infections non invasives à pneumocoque, comme l'otite moyenne aiguë (OMA), la sinusite et la bronchite ainsi que leurs complications, n'étaient pas incluses. Ces infections représentent des motifs fréquents de recours aux soins en Tunisie. Pour les OMA, par exemple, le nombre d'épisodes par enfant et par an est estimé à 0,436 (35), dont environ $35 \%$ sont dues au pneumocoque (36).

En présence d'un vaccin dont le prix est relativement élevé mais qui a démontré une efficacité protectrice de
$97 \%$ contre les infections invasives à pneumocoque (3) et de 52,6\% contre les OMA à pneumocoque (4), une analyse plus poussée des données de l'étude, entrant dans le cadre d'une analyse coût-efficacité, s'avère nécessaire pour la prise de décision. Dans ce cadre, des études d'analyse coût-efficacité ont montré une réduction des coûts après utilisation du vaccin anti-pneumococcique par rapport au coût de prise en charge de la maladie (37). Un effet réducteur de l'incidence de la survenue de la maladie serait également observé suite à l'usage de la vaccination (38).

Toutefois, il faut insister sur le fait que l'utilisation du vaccin anti-pneumococcique doit être considérée comme complémentaire à d'autres interventions essentielles pour lutter contre les infections à pneumocoques et en particulier celles des voies respiratoires, notamment la promotion de l'allaitement maternel exclusif pendant les six premiers mois de la vie, une nutrition adéquate et le recours aux antibiotiques pour la prise en charge des cas (2).

\section{Remerciements}

Les auteurs du présent article tiennent à remercier le personnel du service financier de l'hôpital Fattouma Bourguiba de Monastir et en particulier M. Tamboura Amara, le personnel du service Pharmacie de l'hôpital et son chef de service ainsi que les autres personnes ayant contribué à la collecte des données de cette étude, à savoir Dr Khalsi Fatma, Dr Nouira Nessrine, Dr Khelif Tarek, Dr Mnasri Syrine, Dr Mattoussi Nadia, Dr Ben Ameur Houssine, Dr Gargouri Lamia, Dr Hammami Olfa, Dr Rhayem Siwar, Dr Kechrid Amel et Dr Mezghani Sonda. Un hommage particulier doit être rendu à l'une des sommités de la médecine préventive et communautaire en Tunisie, le Professeur Mohamed Soussi Soltani, qui a coordonné ce projet et a veillé à communiquer ses résultats aux autorités sanitaires tunisiennes durant ses derniers jours de lutte ultime contre sa maladie, ce qui est d'autant plus héroïque qu'elle était tenue secrète. Que son âme repose en paix.

Financement : L'étude a été en partie financée par les Laboratoires Pfizer.

Conflits d'intérêt : Les Laboratoires Pfizer ont certes contribué au financement de ce projet, mais ceci s'est limité à la mise à disposition de l'Hôpital universitaire de Monastir d'une somme d'argent au profit de cette étude, qui a servi à fournir des primes incitatives aux différents intervenants dans le déroulement de l'enquête. Les représentants des laboratoires n'ont eu aucune relation directe avec les personnes qui ont assuré la collecte et l'analyse des données ni avec celles qui ont réalisé le calcul du coût. À la fin, une copie du rapport final de l'étude a été envoyée aux Laboratoires Pfizer. 


\title{
Hospital cost of invasive pneumococcal disease in children aged under 15 years old in Tunisia
}

\begin{abstract}
Background: Pneumococcal infections are an important cause of morbidity and mortality in the world and in Tunisia. Data on the economic burden of these infections are needed to inform decision-making to include pneumococcal vaccinations in routine childhood immunization.
\end{abstract}

Aims: This study aimed to estimate the medical cost of hospitalizations due to invasive pneumococcal disease (pneumonia and meningitis) among children aged under 15 years old in Tunisia.

Methods: A prospective multicentre study was conducted in 15 paediatric departments, across different socio-economic areas of Tunisia, from June 2014 to May 2015. All children aged under 15 years old who were hospitalized for pneumococcal pneumonia or confirmed bacterial meningitis were enrolled. A case report form was completed for every eligible case. Activity Based Costing method was used to estimate the hospital cost. Data entry and statistical analysis were conducted using SPSS, version 20.0 .

Results: During the study period, 727 children were hospitalized for pneumococcal pneumonia and 60 children were hospitalized for bacterial meningitis, among them $21(35 \%)$ had confirmed pneumococcal meningitis. The median hospital cost for pneumococcal pneumonia was 353.910 Tunisian Dinars (TND) and TND 1680.632 for pneumococcal meningitis. Using overall data extrapolation, it was estimated that nearly 1091 hospitalizations for pneumococcal pneumonia and 69 hospitalizations for pneumococcal meningitis occurred each year in Tunisian children aged under 15 years of age, incurring total costs of TND 502 079.408.

Conclusion: The economic burden of pneumococcal infections seems to be substantial in Tunisia. The estimated costs does not reflect the real costs of this infection. Cost-effectiveness studies would be helpful to inform policy-makers to take appropriate decisions.

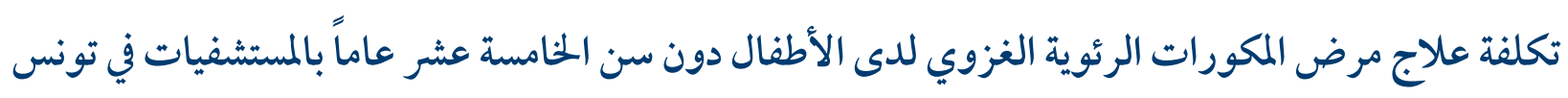

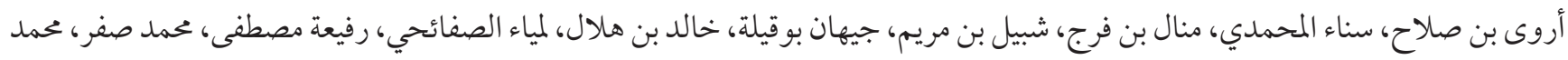

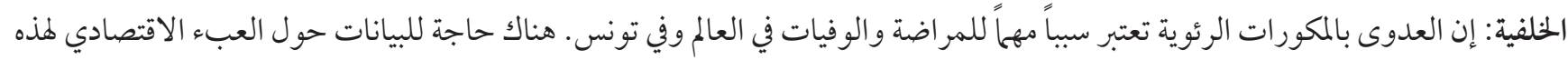

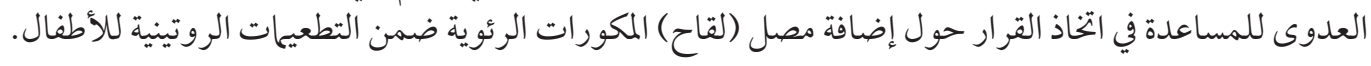

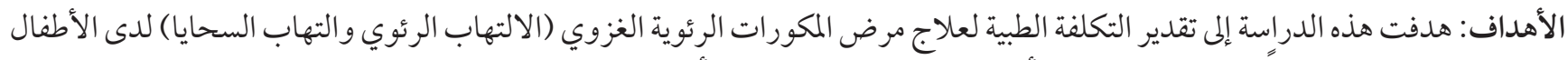

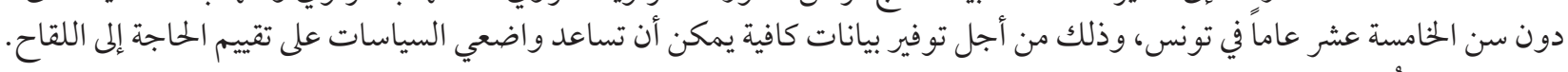

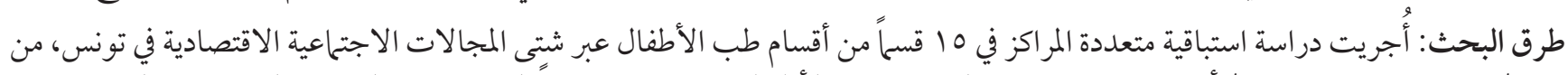

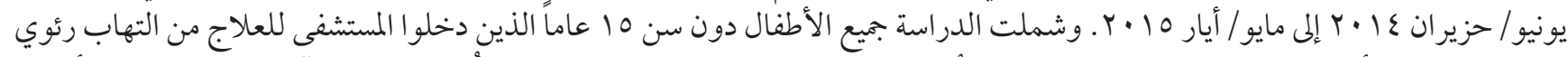

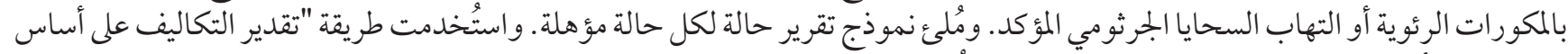

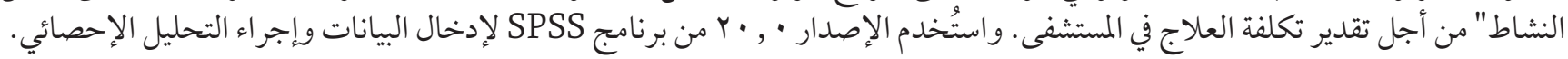

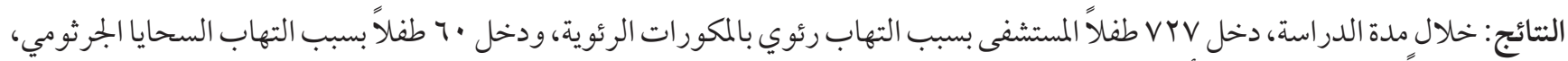

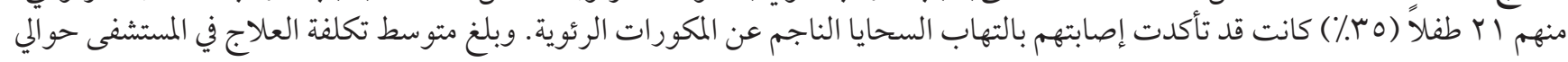

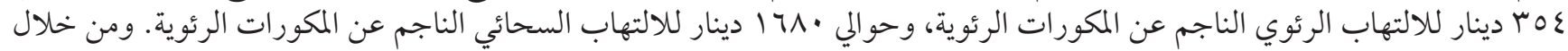

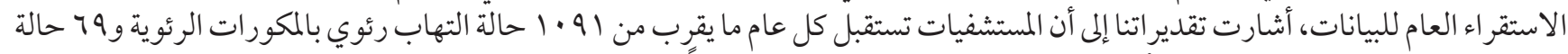

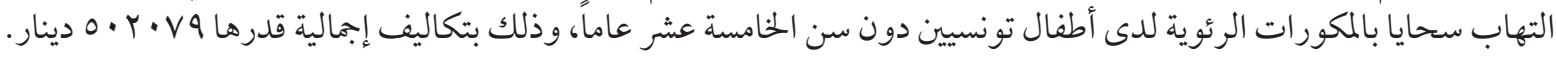

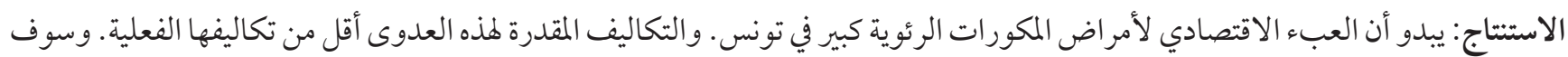

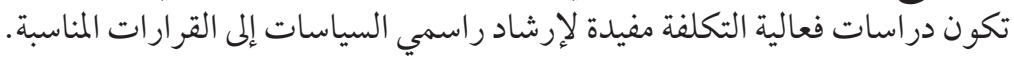




\section{Références}

1. O’Brien KL, Wolfson LJ, Watt JP, Henkle E, Deloria- Knoll M, McCall N, et al. Burden of disease caused by Streptococcus pneumoniae in children younger than 5 years: global estimates. Lancet. 2009 Sep 12;374(9693):893-902. https://doi. org/10.1016/S0140 - 6736(09)61204 - 6 PMID:19748398

2. World Health Organization. Pneumococcal vaccines. WHO position paper - 2012. Wkly Epidemiol Rec. 2012;87(14):129-44.

3. Black S, Shinefield H, Fireman B, Lewis E, Ray P, Hansen JR, et al. Efficacy, safety and immunogenicity of heptavalent pneumococcal conjugate vaccine in children. Northern California Kaiser Permanente Vaccine Study Center Group. Pediatr Infect Dis J. 2000 Mar;19(3):187-95. https:/doi.org/10.1097/00006454 - 200003000 - 00003 PMID:10749457

4. Prymula R, Peeters P, Chrobok V, Kriz P, Novakova E, Kaliskova E, et al. Pneumococcal capsular polysaccharides conjugated to protein D for prevention of acute otitis media caused by both Streptococcus pneumoniae and non - typable Haemophilus influenzae: a randomised double-blind efficacy study. Lancet. 2006 Mar 4;367(9512):740-8. https://doi.org/10.1016/S0140 6736(06)68304 - 9 PMID:16517274

5. Vesikari T, Wysocki J, Chevallier B, Karvonen A, Czajka H, Arsène JP, et al. Immunogenicity of the 10 - valent pneumococcal non - typeable Haemophilus influenzae protein D conjugate vaccine (PHiD - CV) compared to the licensed 7vCRM vaccine. Pediatr Infect Dis J. 2009 Apr;28(4 Suppl):S66-76. https://doi.org/10.1097/INF.0b013e318199f8ef PMID:19325449

6. Bermal N, Szenborn L, Chrobot A, Alberto E, Lommel P Gatchalian S, et al. The 10 -valent pneumococcal non - typeable Haemophilus influenzae protein D conjugate vaccine (PHiD - CV) coadministered with DTPw - HBV/Hib and poliovirus vaccines: assessment of immunogenicity. Pediatr Infect Dis J. 2009 Apr;28(4 Suppl):S89-96. https://doi.org/10.1097/ INF.0b013e318199f901 PMID:19325451

7. Chevallier B, Vesikari T, Brzostek J, Knuf M, Bermal N, Aristegui J, et al. Safety and reactogenicity of the 10 - valent pneumococcal non - typeable Haemophilus influenzae protein D conjugate vaccine (PHiD - CV) when coadministered with routine childhood vaccines. Pediatr Infect Dis J. 2009 Apr;28(4 Suppl):S109-18. https://doi.org/10.1097/ INF.0b013e318199f62d PMID:19325447

8. Esposito S, Tansey S, Thompson A, Ferrera G, Maida A, Bona G, et al. [online]. Safety and immunologic non - inferiority of 13 - valent pneumococcal conjugate vaccine given as a 3 - dose series with routine vaccines in healthy children. Pediatr Infect Dis J. 2009;28(6);e169.

9. Sibak M, Moussa I, El-Tantawy N, Badr S, Chaudri I, Allam E, et al. Cost-effectiveness analysis of the introduction of the pneumococcal conjugate vaccine (PCV - 13) in the Egyptian national immunization program, 2013. Vaccine. 2015 May 7;33 Suppl 1:A182-91. https://doi.org/10.1016/j.vaccine.2014.12.044 PMID:25919159

10. Khemiri M. Particularités des infections à pneumocoque chez l'enfant et leur prévention. 2010 (https://www.infectiologie.org. tn/pdf_ppt_docs/cmi/tunis300610/khemiri.pdf, consulté le 15 mars 2019)

11. Khalifa AB, Mastouri M, Abdallah HB, Noomen S, Kheder M. Les méningites purulentes dans la région de Monastir, Tunisie (1999-2006): aspects bactériologiques et état de résistance aux antibiotiques. Bull Soc Pathol Exot. 2011 Feb;104(1):42- 8. https://doi.org/10.1007/s13149-010 - 0077- 5 PMID:20835902

12. Maalej SM, Kassis M, Rhimi FM, Damak J, Hammami A. Bactériologie des méningites communautaires dans la région de Sfax, Tunisie (1993 - 2001). Med Mal Infect. 2006 Feb;36(2):105-10. https://doi.org/10.1016/j.medmal.2005.10.011 PMID:16459040

13. Werno AM, Murdoch DR. Medical microbiology: laboratory diagnosis of invasive pneumococcal disease. Clin Infect Dis. 2008 Mar 15;46(6):926-32. https://doi.org/10.1086/528798 PMID:18260752

14. Dragos Z. Genèse et évolutions d'une innovation : la méthode ABC. 2010. (https://halshs.archives - ouvertes.fr/ halshs - 00460128/document, consulté le 15 mars 2019).

15. Briciu S, Căpuşneanu S. Effective cost analysis tools of the activity-based costing $(A B C)$ method. Annales Universitatis Apulensis Series Oeconomica. 2010;12(1):25-35.

16. Arrêté du ministre de la santé publique du $1^{\mathrm{er}}$ juin 2006, fixant la nomenclature générale des actes professionnels des médecins, biologistes, médecins dentistes, psychologues cliniciens, sages-femmes et auxiliaires médicaux. Journal Officiel de la République Tunisienne. 2006;46:1514-7 (http://www.legislation.tn/sites/default/files/fraction - journal - officiel/2006/2006F/046/ TF20062734.pdf, consulté le 21 avril 2019).

17. République Tunisienne. Ministère de la Santé. Tarifs de soins dans les structures sanitaires publiques (arrêté du 19 décembre 1996) - Tarifs des lettres clés des actes professionnels relatifs aux payants (http://www.santetunisie.rns.tn/fr/ prestations/tarifications-des-prestations-hospitalieres, consulté le 4 avril 2019).

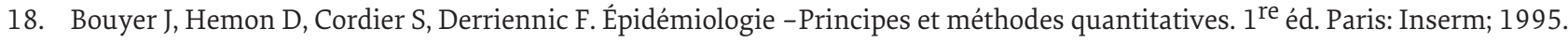

19. Institut National de la Statistique (INS). Indicateurs démographiques (http://www.ins.tn/fr/themes/population, consulté le 4 avril 2019).

20. Griveaux JM. Pilotage de l'activité par les coûts : application de la méthode A.B.C. en stérilisation [thèse]. Nancy, Université Henri Poincaré - Nancy, 2002.

21. Principaux indicateurs du développement social en Tunisie. Tunis : Ministère des Affaires sociales, Bureau des Études, de la Planification et de la Programmation; 2012 (http://www.social.gov.tn/fileadmin/user1/doc/PRINCIPAUX_INDICATEURS_DE_ DEVELOPPEMENT2012 - fr.pdf, consulté le 25 mars 2019). 
22. Institut National de la Statistique. Enquête nationale sur le budget, la consommation et le niveau de vie des ménages, 2015 (http://www.ins.tn/sites/default/files/publication/pdf/consommation2016 - V5 - corrige\%CC\%81.pdf, consulté le 6 avril 2019 ) [en arabe].

23. Ben Gobrane H, Aounallah - Skhiri H, Oueslati F, Frikha H, Achour N, Hsairi M. Estimation du coût de la prise en charge du cancer invasif du col de l'utérus en Tunisie. Sante Publique. 2009 Nov-Dec;21(6):561-9. https://doi.org/10.3917/ spub.096.0561

24. Brotons P, Gelabert G, Launes C, Sicuri E, Pallares R, Muñoz-Almagro C. Cost of hospitalizing children with invasive pneumococcal pneumonia. Vaccine. 2013 Feb4;31(7):1117-22. https://doi.org/10.1016/j.vaccine.2012.12.025 PMID:23262168

25. Milne RJ, Vander Hoorn S. Burden and cost of hospital admissions for vaccine - preventable paediatric pneumococcal disease and non-typable Haemophilus influenza otitis media in New Zealand. Appl Health Econ Health Policy. 2010;8(5):281-300. https://doi.org/10.2165/11535710-000000000-00000 PMID:20804222

26. Colombo GL. [Cost - of - illness study of pneumococcal disease in Italian children]. Ann Ig. 2002 Sep - Oct;14(5):373-88 [In Italian]. PMID:12508446

27. Huang SS, Johnson KM, Ray GT, Wroe P, Lieu TA, Moore MR, et al. Healthcare utilization and cost of pneumococcal disease in the United States. Vaccine. 2011 Apr 18;29(18):3398-412. https://doi.org/10.1016/j.vaccine.2011.02.088 PMID:21397721

28. Statistiques mondiales. États-Unis [Internet]. (http://www.statistiques - mondiales.com/etats_unis.htm, consulté le 15 mars 2019).

29. Constenla D. Evaluating the costs of pneumococcal disease in selected Latin American countries. Rev Panam Salud Publica. 2007 Oct;22(4):268-78. PMID:18078589

30. Alvis Guzmán N, de la Hoz Restrepo F, Higuera AB, Pastor D, Di Fabio JL. Costos económicos de las neumonías en niños menores de 2 años de edad, en Colombia [The economic costs of pneumonia in children under 2 years of age in Colombia]. Rev Panam Salud Publica. 2005;17(3):178-83. PMID:15826397

31. Lagos R, Muñoz A, Espinoza A, Dowes A, Ruttimann R, Colindres R, et al. Costos médicos directos de enfermedades neumocócicas invasores y neumomías con diagnóstico radiológico en niños chilenos. [Direct medical costs of invasive pneumococcal disease and radiologically - diagnosed pneumonia among Chilean children]. Rev Panam Salud Publica. 2009 Aug;26(2):101-11. PMID:19814889

32. Bahia L, Toscano CM, Takemoto ML, Araujo DV. Systematic review of pneumococcal disease costs and productivity loss studies in Latin America and the Caribbean. Vaccine. 2013 Jul 2;31 Suppl 3:C33-44. https://doi.org/10.1016/j.vaccine.2013.05.030 PMID:23777689

33. Banque mondiale. RNB par habitant, méthode Atlas (\$ US courants) [Internet]. (https://donnees.banquemondiale.org/indicateur/ ny.gnp.pcap.cd, consulté le 15 mars 2019).

34. Hussain H, Waters H, Omer SB, Khan A, Baig IY, Mistry R, et al. The cost of treatment for child pneumonias and meningitis in the Northern Areas of Pakistan. Int J Health Plann Manage. 2006 Jul - Sep;21(3):229-38. PMID:17044548

35. Enquête nationale sur la santé et le bien-être de la mère et de l'enfant (Multiple Indicator Cluster Survey [MICS] II-2000). Tunis : Ministère de la Santé publique, Direction des Soins de Santé de Base/Fonds des Nations Unies pour l'Enfance (UNICEF) ; 2000.

36. Musher DM. Streptococcus pneumoniae. In: Mandell GL, Bennett JE, Dolin, eds. Mandell, Douglas, and Bennett's Principles and Practice of Infectious Diseases, 5th ed. Philadelphia: Churchill Livingstone; 2000:2128-47.

37. Boccalini S, Bechini A, Levi M, Tiscione E, Gasparini R, Bonanni P. Cost - effectiveness of new adult pneumococcal vaccination strategies in Italy. Hum Vaccin Immunother. 2013 Mar 1;9(3):699-706. https://doi.org/10.4161/hv.23268 PMID:23295824

38. de Wals P, Black S, Borrow R, Pearce D. Modeling the impact of a new vaccine on pneumococcal and nontypable Haemophilus influenzae diseases: a new simulation model. Clin Ther. 2009 Oct;31(10):2152-69. https://doi.org/10.1016/j. clinthera.2009.10.014 PMID:19922887 